\title{
Rodrigo Vergara Ruiz Maestro de la enseñanza y la divulgación entomológica
}

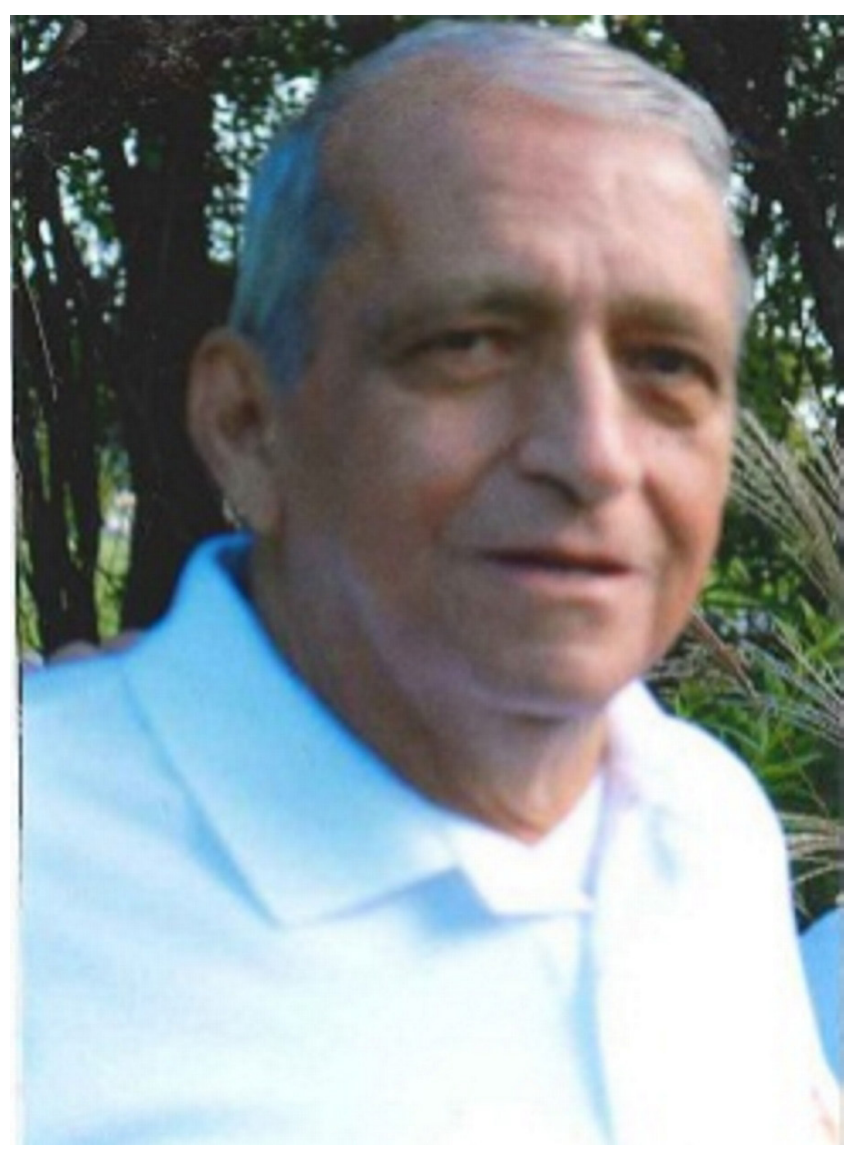

Profesor Rodrigo A. Vergara R.

"Cuando un amigo se va queda un espacio vacio que no lo puede llenar la llegada de otro amigo”.

Alberto Cortés (Cantautor argentino)

El 28 de diciembre de 2017 falleció en la ciudad de Medellín, el distinguido Ingeniero Agrónomo, profesor universitario, entomólogo, colega y apreciado amigo Rodrigo Antonio Vergara Ruiz (Fig. 1).

El profesor Vergara nació en Anorí (Antioquia) el 17 de mayo de 1945. Cursó su bachillerato en el Liceo Antioqueño anexo a la Universidad de Antioquia. Recibió su título profesional de Ingeniero Agrónomo el 12 de febrero de 1971, en la Universidad Nacional de Colombia, sede Medellín.

Adelantó sus estudios de posgrado en Colombia y obtuvo los siguientes títulos: Magister Scientiae en Entomología (Programa de Estudios para Graduados (PEG). ICA-Universidad Nacional de Colombia - Bogotá) y Especialista en Docencia de la Biología (Universidad del Tolima - Ibagué: 1991-1992).

\section{Actividades docentes y administrativas}

$\checkmark$ Profesor universitario de la Facultad de Agronomía, Universidad Pedagógica y Tecnológica de Colombia U.P.T.C (1971 a 1988).

$\checkmark$ Profesor visitante en la Universidad del Tolima (1988 a 1989).

$\checkmark$ Profesor titular en la Universidad del Tolima (1989 a 1992).

$\checkmark$ Secretario Facultad de Agronomía, en la U.P.T.C., Tunja (1976).

$\checkmark$ Representante profesoral al Consejo de Facultad, en la U.P.T.C. y ante los Comités: Docente, de Admisiones y Registros, Curricular y Consejo Superior Universitario de la U:P:T:C:

$\checkmark$ Director del Centro de Investigaciones Agropecuarias INIAG-UPTC (1981-1984) y de la Oficina Central de Investigaciones de la Universidad del Tolima (1990 - 1992) y del Departamento de Producción y Sanidad Vegetal (1990) Universidad del Tolima. Director del Departamento de Agronomía. Facultad de Ciencias Agropecuarias de la Universidad Nacional de Colombia, sede de Medellín (1993 -1994).

$\checkmark$ Profesor Asociado. Departamento de Agronomía. Universidad Nacional de Colombia, sede de Medellín. Facultad de Ciencias Agropecuarias. (1992 a 2004) (Fig. 1).

$\checkmark$ Decano Facultad de Ciencias Agropecuarias. Universidad Nacional de Colombia, sede de Medellín. (Abril de 1994 hasta abril de 1996).

$\checkmark$ Vicepresidente de la Asociación de Profesores Universidad, ASPU, 1981-1982.

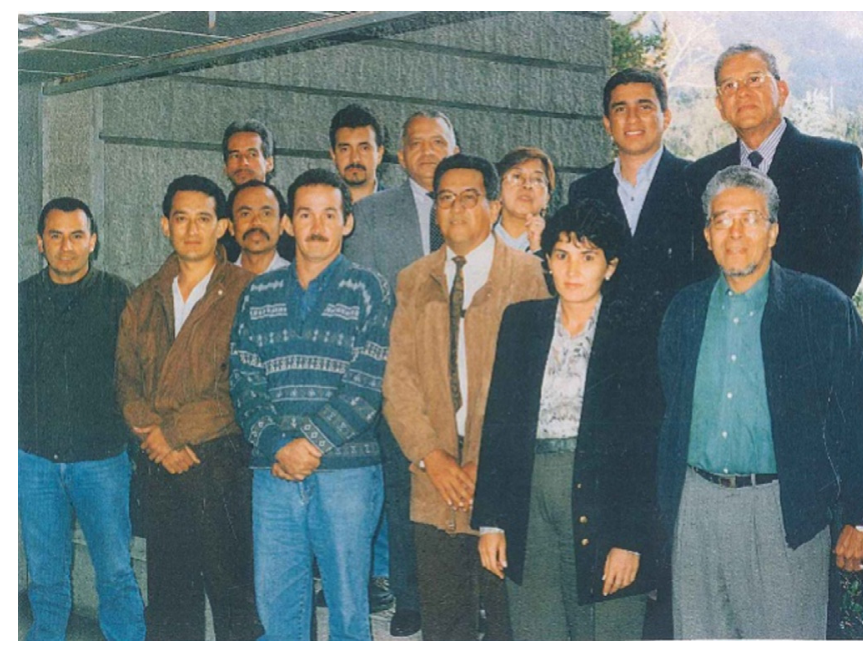

Figura 1. El profesor Rodrigo A. Vergara R. con el grupo de docenes participantes en el "Encuentro Nacional de Profesores de Entomología" celebrado en la Universidad Javeriana en Bogotá. 


\section{Actividades de Extensión Universitaria}

El profesor Rodrigo Vergara realizó un importante aporte en el campo de la Extensión Universitaria y de la asesoría fitosanitaria en condiciones de campo. Como tal, fue un activo promotor y conferencista en diferentes eventos tales como Simposios, Congresos de Entomología y Ecología, Foros, y también de Talleres y Seminarios Entomológicos, con énfasis en manejo de plagas de importancia económica.

Conviene mencionar como ejemplo de lo anterior, su activa participación en los Congresos Anuales de la Sociedad Colombiana de Entomología- SOCOLEN, en los Simposios Nacionales de Control Biológico, en los Foros Entomológicos de la Facultad de Ciencias Agropecuarias, sede Palmira, en los Simposios Internacionales y Nacionales sobre el impacto de los plaguicidas en la salud humana y el ambiente, organizados por Rapalmira y la Universidad Nacional de Colombia - sede Palmira. Promovió en unión de profesores y estudiantes de la Universidad Nacional sede Medellín, los tradicionales Seminarios anuales del GEUN (Fig. 2).

Fue además miembro permanente del Consejo Regional de Plaguicidas de Antioquia y ponente en sus respectivos seminarios. Participó como conferencista en los dos primeros eventos de "Insectópolis" efectuados en la ciudad de Cali. Fue integrante del Comité Nacional de Control Biológico, grupo auspiciado por Colciencias. Igualmente el profesor Vergara promovió talleres y jornadas de campo con comunidades de agricultores en diferentes regiones de Antioquia, sobre el manejo racional de plagas agrícolas con un enfoque MIP; esto lo desarrolló conjuntamente con un equipo de profesores de la Universidad Nacional y de profesionales de Cornare, principalmente en el Oriente Antioqueño (Fig. 3).

Se destaca también la publicación continua que el profesor Vergara realizó de interesantes artículos entomológicos en revistas divulgativas como Metroflor (Bogotá) y también artículos científicos y libros de carácter universitario referentes al manejo integrado de plagas agrícolas. Complementariamente se desempeñó como autorizado asesor fitosanitario de diferentes empresas agropecuarias nacionales y de insumos biológicos (Productos Biológicos Perkins - Palmira). También fue consultor permanente de agremiaciones de flo-

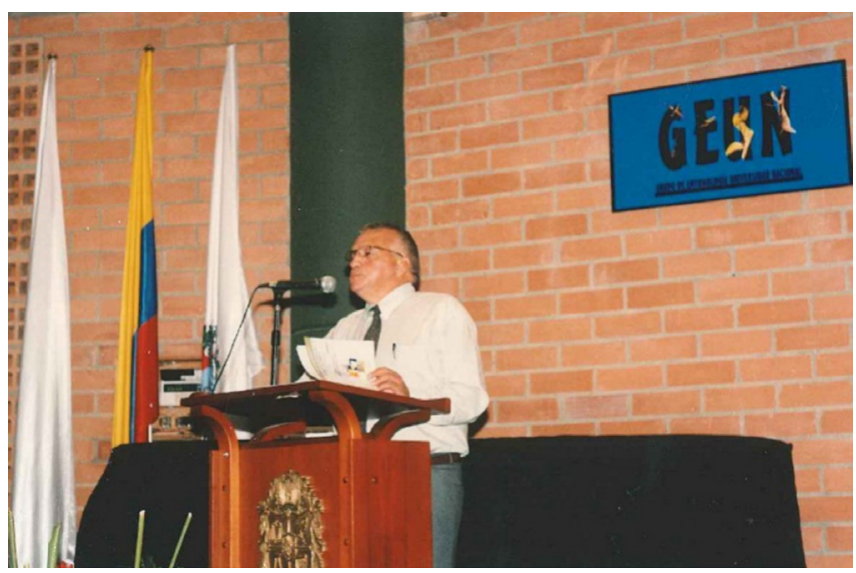

Figura 2. El Dr. Rodrigo A. Vergara R. instalando el seminario de "Aconteceres Entomológicos", organizado por el "GEUN- Grupo de Entomología de la Universidad Nacional- sede Medellín, del cual fue gestor, en compañía del profesor Francisco C. Yepes y de sus estudiantes en los cursos de entomología.

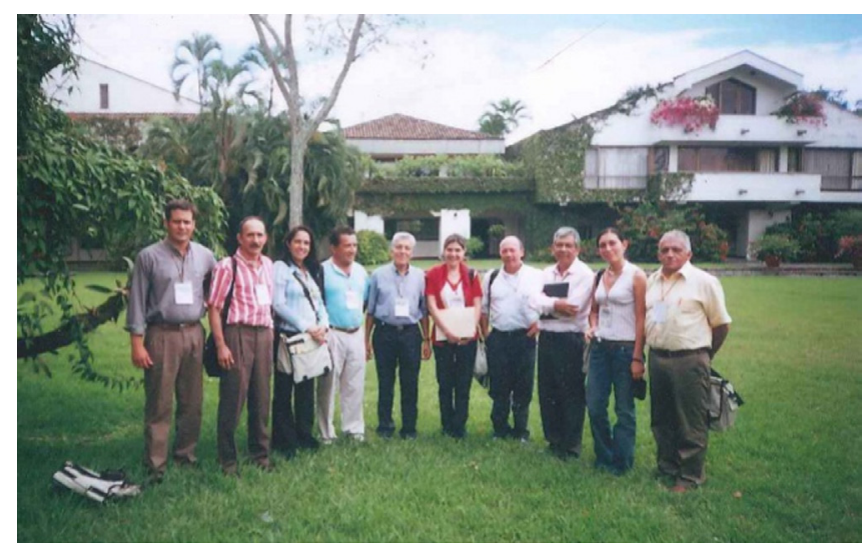

Figura 3. El profesor Rodrigo A. Vergara R. con entomólogos asistentes al "Congreso Internacional de Control Biológico", realizado en el CIAT- Palmira.

ricultores, arroceros, paperos, fruticultores, algodoneros y cerealeros.

\section{Profesor visitante en el exterior}

Escuela Politécnica del Ejército. Facultad de Ciencias Agropecuarias. Maestría en Ciencias del Control Biológico, IASA, San Golqui, Ecuador.

\section{Premios y distinciones}

$\checkmark$ Resolución Consejo de la Facultad de Agronomía U.P.T.C., abril 17 de 1980. Por lo cual se hace una distinción a la capacidad investigativa y se le reconoce como ejemplo para la juventud.

$\checkmark$ Dedicación de dos nuevas especies de insectos por taxónomos de Checoeslovaquia y Estados Unidos:

1) Scrobipalpopsis (Scrobischema subgen. n.) vergarai sp. n., a potential pest species in Colombia (LepidopteraGelechiidae). "Acta Entomológica Bohemoslovaca" 77: 53-63. 1980. Autor: Dalibor Povolny.

2) The species of the Genus Suilla found in the American South the United States (Diptera: Heleomyzidae). Suillia vergarae Steyskal. "Proc. Entomol. Soc. Wash" 1980: 401-404.

$\checkmark$ Moción y diploma de la Asociación Colombiana de Ciencias Biológicas, A.C.C.B. Primer premio en el Congreso Nacional de A.C.C.B. 1983.

$\checkmark$ Ingeniero Agrónomo del Año en Boyacá. 1985 (Medalla y placa). Otorgado por SIABOY, Sociedad de Ingenieros Agrónomos de Boyacá.

$\checkmark$ Mención de Honor de la Sociedad de Ingenieros Agrónomos del Huila. XII Congreso Nacional de Ingenieros Agrónomos. Neiva, 1987, por la labor al frente del gremio.

$\checkmark$ Logo-símbolo de la Universidad del Tolima, por ascenso a profesor titular. Entrega del Escudo en Ceremonia Especial.

$\checkmark$ Protector de la Naturaleza. Reconocimiento y mención del Instituto Dominicano de Bio- Conservación. Santo Domingo-República Dominicana.

$\checkmark$ Mención de Honor - Consejo Directivo Facultad de Ciencias Agropecuarias - U.N. Palmira- Octubre de 1995. En calidad de Profesor Adscrito.

$\checkmark$ Docencia Excepcional de la Universidad Nacional de Colombia, 1995, 1996, 1997, 1999. Premio al mejor docente 
de la Facultad que otorga el Consejo Superior Universitario de la Universidad Nacional de Colombia, cada año.

$\checkmark$ Proyecto de Extensión Solidaria de la Universidad Nacional de Colombia, 1997.

$\checkmark$ Premio único al proyecto ejecutado en el área de capacitación a técnicos y agricultores en el empleo de estrategias de Manejo Integrado de Plagas que sustituyan el uso de plaguicidas. Otorga el Consejo Superior Universitario de la Universidad Nacional de Colombia.

$\checkmark$ Distinción de Honor "Al Maestro". Asociación de Profesores Universitarios de Antioquia y Universidad Nacional de Colombia, sede de Medellín. Recibió "La Pluma de Ganso" y Diploma Especial 2001. Por el trabajo como educador por más de 30 años.

$\checkmark$ Medalla al Mérito. Liga Colombiana de Consumidores por "La defensa del medio ambiente y la lucha contra plaguicidas". Medellín, Noviembre de 2001.

$\checkmark$ Premio "Héctor Delgado Zambrano" de la Sociedad Colombiana de Entomología- SOCOLEN, a la vida y obra de un investigador de la Entomología en Colombia. Julio de 2004.

$\checkmark$ Placa Conmemorativa - Octubre 27 de 2010. Otorgada por el Comité Regional de Plaguicidas del Oriente Antioqueño, en sus 20 años como reconocimiento al profesor Rodrigo Vergara Ruiz, por sus valiosos aportes académicos y científicos para el desarrollo sostenible del sector agropecuario del país.

\section{Publicaciones}

$\checkmark 193$ artículos entomológicos en revistas científicas, tecnológicas y divulgativas.

$\checkmark \quad 9$ libros universitarios sobre temas básicos y manejo de plagas agrícolas, además de importantes ensayos sobre el impacto de los plaguicidas en la salud y el ambiente.

$\checkmark$ Dirección de 100 trabajos de grado y de tesis de posgrado en las áreas de la Entomología agrícola básica y aplicada.

\section{Membresía}

$\checkmark$ Socio fundador No. 64 de la Sociedad Colombiana de Entomología - SOCOLEN.

$\checkmark$ Socio fundador del Grupo de Entomología de la Universidad Nacional de Colombia, Sede de Medellín - GEUN

$\checkmark$ Miembro adherente de: National Geographic Society USA.

\section{Algunos rasgos personales del maestro Rodrigo:...}

$\checkmark$ El maestro Rodrigo Vergara Ruiz era supremamente estricto con sus estudiantes y no perdonaba la llegada tarde o la falta a clases. Muy rígido en las calificaciones, sin ser un tirano. Siempre agregaba unas décimas a los que perdían el examen y les advertía a quienes le solicitaban la revisión de la nota, que si se sometían a este proceso, corrían el riesgo de obtener otra menor. Frente a esta posibilidad, la mayoría de sus discípulos no hacía reclamos.

$\checkmark$ No escatimaba sacrificios frente a sus estudiantes, quienes ante la actitud perseverante del docente y el ánimo de dar más de lo exigido por la institución, solo tenían la alternativa de mejorar su rendimiento académico y mascullar en solitario la ira santa. Actitud que cambiaba diametralmente cuando recibían su título profesional, momento en el cual no sabían que hacer con este genio de la educación entomológica, expresando públicamente el honor de haber tenido semejante maestro.

$\checkmark$ Finalmente cabe destacar que para el profesor Rodrigo Vergara Ruiz, paralelo a sus actividades universitarias en docencia, investigación y extensión y en cargos académico-administrativos, fue siempre muy importante su núcleo familiar. En efecto, se esmeró por brindarles a sus hijos lo mejor en valores humanos, en formación académica y en calidad de vida, todo ello en compañía de su fiel y diligente esposa Alcira, preocupados ambos por el bienestar familiar centrado como prioridad en sus tres hijos: José Daniel, Ingeniero Agrónomo, Diana Lucía, Ingeniera Ambiental y Carlos, Administrador Agropecuario. Y en su condición de abuelo siempre manifestó un desbordado cariño para con sus nietos Lucas, Antonia e Isabela. Igualmente Rodrigo gozó de un cálido y permanente afecto de parte de sus 9 hermanos y hermanas. Y por supuesto es de resaltar que para quienes lo conocimos de cerca y tuvimos el privilegio de disfrutar de su amistad y compañía, no dudamos en calificar a Rodrigo como un excelente amigo y un brillante profesional de las Ciencias Agronómicas y Entomológicas.

Por: José Iván Zuluaga C. y Francisco C. Yepes R. Profesores Universidad Nacional de Colombia joseivanzuluagac@hotmail.com fcyepes@unal.edu.co 